\title{
Effect of model simplification through manual reduction in number of surfaces on room acoustics simulation
}

\author{
Nurul Amira Abd Jalil ${ }^{1}$, Nazli Bin Che Din ${ }^{1,2^{*}}$, Nila Keumala ${ }^{1}$, Asrul Sani Razak ${ }^{1,2}$ \\ ${ }^{I}$ Department of Architecture, Faculty of Built Environment, University of Malaya \\ ${ }^{2}$ The Centre for Building, Construction \& Tropical Architecture (BuCTA), Faculty of Built Environment, \\ University of Malaya \\ nazlichedin@um.edu.my ${ }^{{ }^{*}}$
}

Received: $29^{\text {th }}$ September $2018 \quad$ Final version received:13 $3^{\text {th }}$ December 2019

\begin{abstract}
Model simplification is an important step in room modelling for acoustics simulation. Various simplification algorithms were previously suggested. However, they are highly demanding methods, which are more suitable for large complicated spaces. As this paper focused on small size open-plan office (OPO) spaces, basic modelling and manual simplification processes were used. The aim is to find the optimum level of model simplification through a manual reduction in number of surfaces. Parallel to this aim, the objective is to examine the effects of the change in model detail level on selected acoustical parameters in six OPOs. Construction of models started with the most detailed model. Simplified models were developed by gradually reducing the number of surfaces. Simulation on RT, STI and decay in SPL were done and compared to the measured data. The study found that models with up to $80 \%$ reduction in number of surfaces can still produce plausible simulation results.
\end{abstract}

\section{INTRODUCTION}

Architecture uses visuals as its 'tools of the trade' to convey ideas (J. Schroeder et.al, 1996). Visualization has been the most practical approach of presenting imaginary spaces (A. Koutamanis, 2000). Initially, visual communication in architecture was delivered through hand drawn sketches and drawings. However, this process demands meticulous effort and the process of producing aesthetically appealing visuals is often time consuming.

Nowadays, with the aid of computer technology, architects migrated towards producing their ideas digitally (Ö. Akin, 1990). Using computers, twodimensional design sketches of preliminary ideas can turn into three-dimensional (3D) models in a short amount of time. Besides having the advantages of viewing the space in a 3D viewpoint, these modelling tools can also be used as a medium to explore ideas, forms, shapes, spaces and function (X. Yin et. al, 2009).

In addition to being a medium for architectural visuals, these models can also be utilized for the prediction of building performances (A. Yezioro. 2008). The ability to predict building performance is a potent instrument in the construction industry. Successful planning of building performance can potentially lead to sustainable energy conservation (T. Hong et. al, 2000).

One element in building a performance that can benefit from these 3D models is acoustics. In the past decades, the process of transforming 2D architectural drawings into digital formats for room acoustics evaluation was described as tedious and laborious work. As a result, establishing assessments toward these processes took a lot of time and effort (D. J. Oldham et. al, 1987). But with today' thriving technology development in computer graphics and modelling tools, those stages are considered as the easy ones as 3D models would be readily available since the beginning of the preliminary design stages.

Like other building performance prediction works, acoustics prediction is an important element in the field of built environment (M. Hornikx, 2015) as it can save time, cost, energy, and resources (G. Augenbroe, 2002); especially when the prediction work takes place during the early stages of the design work (S. Siltanen et. al, 2008). However, it was becoming an issue that 
the geometry of digital 3D models created for the purpose of architects' visualization was too complex and often possess irrelevant and thus, redundant geometric details for the purpose of acoustical prediction.

\subsection{Room Acoustics Simulation and Model Simplification}

In room acoustics simulation, model simplification has become a very crucial step in the modelling process. Andújar, Ayala and Brunet described geometry simplification as "generation of 3D models that resemble the input model, but involve fewer faces, edges and vertices." The goal of geometry simplification is to transform an existing model into a simplified model with less complexity while still maintaining the model's essential features (C. Andújar et. al, 2002). As small details contribute no major impact on the simulation result, 3D models with simpler geometry are considered as the more practical medium for room acoustics simulation. This is especially applicable when the wavelength of the sound at mid-frequency of $1000 \mathrm{~Hz}$ is larger than the dimension of the model's surfaces (H. Kuttruff, 2009).

Previous studies proposed some very intricate algorithms in geometry reduction or simplification (F. S. Nooruddin, 2003). The cases presented dealt with models or objects of highly complex geometries. On the other hand, Siltanen et al. presented a geometry reduction process on model of architectural spaces for the purpose of acoustical prediction. Using the combination of topology simplification and surface simplification, the procedure was applied to large spaces such as concert halls and amphitheatres.

Nevertheless, it should be noted that these procedures and algorithms were developed by computer graphics experts and it might be quite challenging for non-experts such as regular architectural 3D designers or architecture students to apply in their modelling work. Moreover, those simplification methods might be well suited for acoustical prediction on projects where the architectural 3D models can be obtained easily from the project principals.

Room acoustics simulation has gone through some rigorous development over the past decade (J. H. Rindel, 2010). This development from merely predicting the acoustical performance of rooms in figures, to the possibility of room auralization has raised interest in acoustic prediction and simulation work from many fields (M. Vorländer, 2014). With the prevalence issue of workplace acoustics and how it affects employees' productivity, motivation, and psychological health (S. A. Ali, 2011), this sensory output through room acoustics simulation can become a channel to raise public awareness on the importance of high-quality workplace acoustics. This effort might just as well work as this type of virtual experience can assist in leaving an impressionable connotation.

At the present time, several numbers of geometrical acoustics simulation software have been introduced. These acoustical simulation tools are commercially available in the market and are widely applied by acoustics consultants as a tool for acoustical prediction (S. Pelzer et. al, 2011). These tools have become reliable and effective in simulating acoustical parameter ( $\mathrm{J}$. H. Rindel, 2000).

ODEON is one of the acoustical simulation software which is available for acoustical prediction. The calculation algorithm utilized in ODEON is a hybrid reflection method that combines the image source method, raytracing method, and ray radiosity together (C. L. Christensen et al, 2005). It is generally easy to use acoustical measurement and prediction tools which is applicable not only for acousticallyessential large spaces such as performance halls, churches, and auditoriums; but also for medium and small spaces like classrooms and open-plan offices (OPOs). The simulation of OPOs spaces was made easier using ODEON when it responded to the recently available ISO 3382$3: 201228$ which is a method specifically for the acoustical measurement in open-plan offices (OPOs).

ODEON software recommended that the surfaces of the model to be built in large dimensions. The basic rule laid out was to keep the surface dimension approximately above 0.34 meters which are larger than one wavelength at the mid frequency (C. L. Christensen et al, 2013). Although this might be possible and maybe even more practical for the construction of auditoriums or large concert hall, it would be rather difficult to keep to the rule when constructing small spaces such as open-plan office (OPO) spaces. Depending on the envelope design of the building and the purpose of the space, OPOs can be very irregular in geometry. 
Unlike auditoriums and concert halls, this irregularity may lead to uneven sound diffusion and with different furniture and office supply settings, sound absorption dispenses unevenly on all surfaces in the room. On the other hand, Shiokawa and Rindel stated that a too simplified model might not bear the correct acoustic behaviour of the space.

Open-plan office spaces used in this paper are existing OPOs which does not have a readily available architectural 3D models that can be simplified using the previously proposed simplification algorithm (S. Siltanen, 2008). Thus, this paper attempts to apply basic modelling techniques for model construction and model simplification process. However, the issue highlighted here is the model detail level. How detailed or simple should the spaces be constructed for the purpose of acoustic simulation using ODEON Room Acoustic Software? And do these discrepancies in the model detail level affect the simulation results of the acoustical parameters? Therefore, the aim of the study is to find the optimum level of model simplification through the simple method of manually reducing the number of surfaces. The objective of the study is to examine the effects of changes in the model detail level through the differences in number of surfaces, on selected acoustical parameters in simulation work.

\section{METHODOLOGY}

\subsection{Model Construction and Simulation}

The rooms modelled and acoustically simulated in this paper are adapted from earlier work. In the previous work, 12 spaces from three green office buildings were measured. Two open-plan offices and two meeting rooms were selected from each building as sample spaces for the measurement of selected acoustical parameters.

Among the 12 spaces measured in the previous study, only open-plan offices (OPOs) were constructed for the purpose of acoustical simulation in this study. This is because the open-plan office layout is one of the most prominent design strategies can be applied in green office building (G.A.Coudriet, 2009) and understandably one of the most important spaces in any office buildings. However, various IEQ studies done in open-plan offices in green office buildings reported a low satisfaction level in acoustic comfort (R. T. Muehleisen, 2006). The acoustics complaints recorded include noise problems related to the lack of sound isolation and the lack of speech privacy (X. Wang et al, 2016).

Furthermore, simulation of OPOs would allow for the comparison of parameters that requires long distances between the sound source and receiver points. Those parameters include speech transmission index (STI) and spatial decay in sound pressure level (SPL), besides the elementary parameter that is reverberation time (RT). Table 1 briefly describes the OPOs adopted in this study.

Table 1. The volume and averaged measured background noise (BN) of the adopted open-plan offices

\begin{tabular}{cccc}
\hline No. & $\begin{array}{c}\text { OPO } \\
\text { Code }\end{array}$ & $\begin{array}{c}\text { Volume } \\
\left(\mathbf{m}^{\mathbf{3}}\right)\end{array}$ & $\begin{array}{c}\text { Averaged } \\
\text { Measured BN, } \\
\mathbf{d B}(\mathbf{A})\end{array}$ \\
\hline Building A & \\
\hline 1 & DOP1 & 756.0 & 30.28 \\
\hline 2 & DOP2 & 846.6 & 36.71 \\
\hline Building B & & \\
\hline 3 & LOP1 & 559.0 & 37.29 \\
\hline 4 & LOP2 & 1035.3 & 31.79 \\
\hline Building C & & 36.33 \\
\hline 5 & GOP2 & 316.8 & 35.0 \\
\hline 6 & GOP2 & 805.2 & \\
\hline
\end{tabular}

As the objective of the study is to examine the effects of model detail level on the selected acoustical parameters, the first step taken was to digitally construct each room based on its layout plan. The construction of the models was carried out using a 3D software modelling tool, SketchUp.

In order to run the acoustical simulation, the models were exported into ODEON Room Acoustic Software. All surfaces were then assigned with materials available in the ODEON material library which suits their estimated characteristics and descriptions. Some materials were not readily available in the ODEON material library and thus needed to be imported from other sources. Sound source and receiver points were placed similarly to the positions established during the physical measurement. The number of receivers and their arrangements varied between the spaces. 
Before the simulation work was carried out, a quick estimation of the reverberation time (RT) was run in order to determine the suitable impulse response length for the calculation. ODEON suggested that the impulse response length should cover at least $2 / 3$ of the calculated RT. Quick estimation showed that the most practical impulse response length to be applied in all models is 2000ms. Table 2 shows the basic room settings information for all rooms. As for the background noise $(\mathrm{BN})$, the averaged octave band frequency from $63 \mathrm{~Hz}$ to $8000 \mathrm{~Hz}$ recorded from selected points during the measurement were entered into the room setup.

Table 2. Basic room information settings fixed in ODEON

\begin{tabular}{lll}
\hline $\begin{array}{l}\text { No. of sound source } \\
\text { Sound source type }\end{array}$ & $: \begin{array}{l}1 \text { (set at } 1.2 \mathrm{~m} \text { high) } \\
\text { OMNI.S08 [Total power: } \\
75.4 \mathrm{~dB}(\mathrm{~A})]\end{array}$ \\
$\begin{array}{l}\text { No. of multi point } \\
\text { receivers }\end{array}$ & $: \begin{array}{l}\text { DOP1, DOP2, LOP1, } \\
\text { LOP2 }-10 \text { points each; } \\
\text { GOP1 } 11 \text { points; GOP2 } \\
-12 \text { points (all set at } 1.2 \\
\text { m high) }\end{array}$ \\
$\begin{array}{l}\text { Impulse response } \\
\text { length }\end{array}$ & $\begin{array}{l}2000 \mathrm{~ms} \\
\text { Temperature input }\end{array}$ & $24^{\circ}$ \\
\hline
\end{tabular}

In defining the primary model for the comparison of model detail level, two models were constructed for each OPO. The first models (model 1) were built as a detail imitation of the real open-plan offices (See Fig. 1). Meanwhile, models 0s were constructed as the most basic models of each OPO. The walls, ceilings, and other surfaces were raised as planes without considering the beams' and columns' intrusions and extrusions. To simplify the presentation and for a better understanding of the readers, only illustrations of models from GOP1 will be presented for the exhibition of the simulation process. Illustration of model 1 and model 0 constructed for GOP1 is shown in Fig. 2.

The initial determination of the most effective model detail level was done by simulating the reverberation time (RT) on both model 1 and model 0 for all the OPOs and comparing them with their respective measured RT. ODEON recommended the subjective limen for RT to be below $5 \%$ of relative difference (C. L. Christensen, 2013).
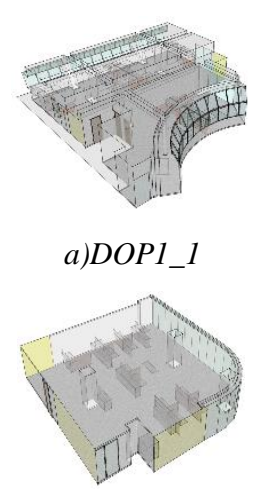

c)LOPI_l

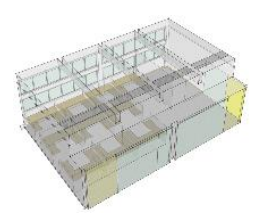

e)GOP1_1

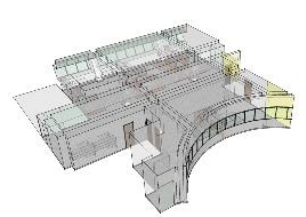

b)DOP2_1

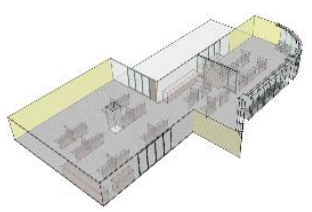

d)LOP2_1

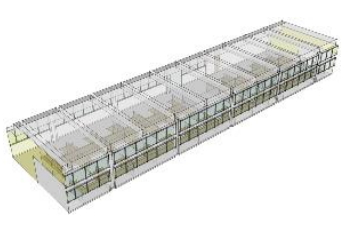

f)GOP2_1
Figure 1: Model 1 for all open-plan offices.

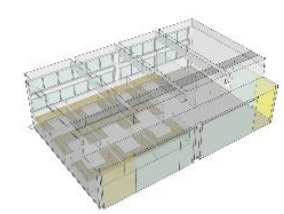

(a) GOP1_1

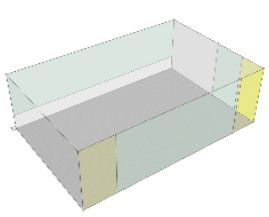

(b) GOP1_O
Figure 2: Model 1 and model 0 of GOP1.

However, Hodgson, and Bistafa and Bradley stated $10 \%$ difference as a more practical maximum relative difference for engineering type accuracy for reverberation time. The relative difference was calculated by comparing the mid-frequency RT (mean from $500 \mathrm{~Hz}$ to 2 $\mathrm{kHz}$ ) of the measured RT value with that of the simulated models. It should be noted that the simulation of RT for all models was done based on the T30 calculation as per done during the physical measurement.

Fig. 3 shows the relative difference between all the OPOs in their respective model 1 and 0 . In general, it is clear that model 1 is the more effective model between the two. Although model 0 of LOP1, LOP2 and GOP1 recorded smaller relative difference and furthermore below the recommended $10 \%$ limit, deduction as a group would determine model 1 as the more reliable model. 


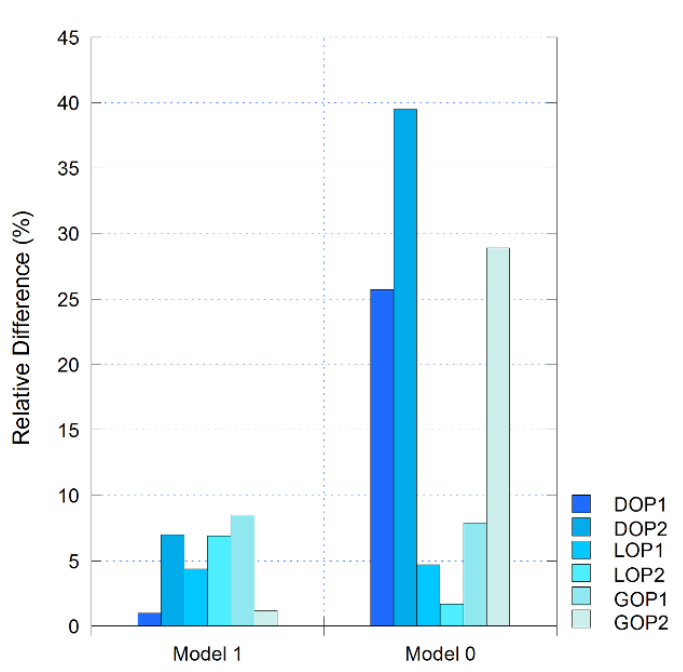

Figure 3: Comparison of relative difference between all OPOs in model 1 and model 0 .

Moving forward, the second stage of the study was developed around the construction of model 1. Model 1 is now determined as the primary model for defining the changes in model detail level and how it would affect the acoustical parameters such as RT, STI and spatial decay of SPL. Based on model 1, two additional models were constructed. Since model 1 was constructed in the most detail imitation of the room's geometry, the additional models were fabricated by gradually reducing the detail level in model 1 , mainly on the structure and building component parts, specifically the doors and windows detailing. However, the furniture layout would stay identical for easy comparison of the changes in detail on the building structure and components only. Fig. 4 shows the additional models of GOP1, which were built based on GOP1_1. Another additional model is a derivation from model 0 from the first stage of the modelling work. However, at this stage; the model was added with the same furniture layout as other models. This is to determine the significance of furniture layout in the simulation process, and to see if basic model envelopes could work well with the addition of furniture layout. At this juncture, model 1 will continue to be identified as model 1 and the other three models will be identified as models 2,3 and 4 . For the purpose of comparison, a duplicate of models 1, 2, 3 and 4 without furniture layout would also be simulated. These duplicates would be identified as models 5, 6, 7 and 8 as illustrated in Fig. 5. It should be noted that model 8 in this stage of the study is, in fact, the same model as model 0 in the first stage of the study.

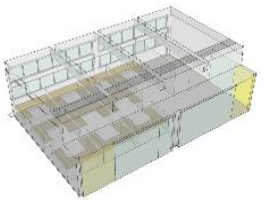

(a) GOPI_l

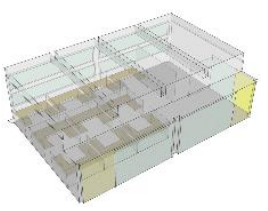

(c) $\mathrm{GOPI}_{-} 3$

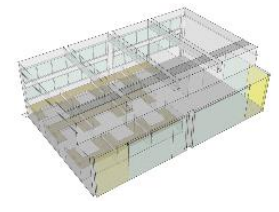

(b) GOPI_2

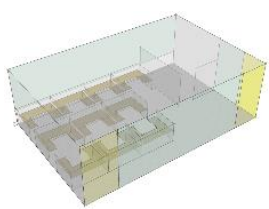

(d) GOPI_4
Figure 4: Model 1, 2, 3, and 4 of GOP1.

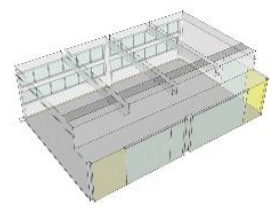

(a) GOPI_5

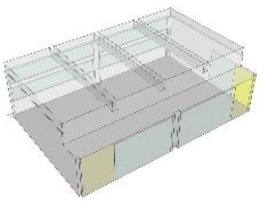

(c)GOP1_7

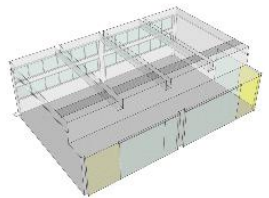

(b)GOP1_6

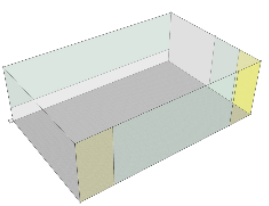

(d)GOP1_8
Figure 5: Duplicate of model 1, 2, 3, and 4 of GOP1 without the furniture.

Table 3. The percentage range of the number of surfaces in model 1, 2, 3, and 4 in all OPOs

\begin{tabular}{|c|c|c|c|c|c|}
\hline \multirow{4}{*}{ OPO } & Model & 1 & 2 & 3 & 4 \\
\cline { 2 - 6 } & $\begin{array}{c}\text { Percentage } \\
\text { Range }\end{array}$ & $100 \%$ & $\begin{array}{c}40- \\
60 \%\end{array}$ & $\begin{array}{c}20- \\
40 \\
\%\end{array}$ & $\begin{array}{c}0- \\
20 \%\end{array}$ \\
\hline \multirow{2}{*}{ DOP1 } & $\begin{array}{c}\text { No. of } \\
\text { surfaces }\end{array}$ & 637 & 305 & 249 & 65 \\
\cline { 2 - 6 } & $\%$ range & 100 & 47.9 & 39.1 & 10.2 \\
\hline \multirow{2}{*}{ DOP2 } & $\begin{array}{c}\text { No. of } \\
\text { surfaces }\end{array}$ & 623 & 295 & 245 & 81 \\
\cline { 2 - 6 } & $\%$ range & 100 & 47.4 & 39.3 & 13.0 \\
\hline \multirow{2}{*}{ LOP1 } & $\begin{array}{c}\text { No. of } \\
\text { surfaces }\end{array}$ & 137 & 72 & 50 & 28 \\
\cline { 2 - 6 } & $\%$ range & 100 & 52.6 & 36.5 & 20.4 \\
\hline \multirow{2}{*}{ LOP2 } & $\begin{array}{c}\text { No. of } \\
\text { surfaces }\end{array}$ & 216 & 116 & 77 & 40 \\
\cline { 2 - 6 } & $\%$ range & 100 & 53.7 & 35.6 & 20.4 \\
\hline \multirow{2}{*}{ GOP1 } & $\begin{array}{c}\text { No. of } \\
\text { surfaces }\end{array}$ & 210 & 96 & 59 & 12 \\
\cline { 2 - 6 } & $\%$ range & 100 & 45.7 & 28.1 & 5.7 \\
\hline \multirow{2}{*}{ GOP2 } & $\begin{array}{c}\text { No. of } \\
\text { surfaces }\end{array}$ & 595 & 238 & 146 & 13 \\
\cline { 2 - 6 } & $\%$ range & 100 & 40.0 & 24.5 & 2.2 \\
\hline
\end{tabular}


Through the modelling process of models 2 and 3 , the percentage of surface number was controlled. This is to ensure that all models from different OPOs to be in the same range for a fair comparison. Table 3 shows the number of surfaces for all OPOs and the controlled range of surface reduction. However, it should be noted that the number of surfaces recorded does not include the number of surfaces from the furniture layout as the furniture layout added to all models for each OPO was constant.

\section{RESULTS AND DISCUSSIONS 3.1. Reverberation Time (RT)}

Fig. 6 shows the relative difference of reverberation time (RT) between the measured and simulated mean RT of $500 \mathrm{~Hz}$ to $2 \mathrm{kHz}$ for all open-plan offices in model 1 until model 8 .

In general, it can be observed that model 5 to 8 from most of the OPOs exceeded the maximum RT relative difference of $10 \%$ applied in this study. However, it should be noted that all models from LOP1 recorded a low relative difference in RT comparison. The same case can be seen for all models in LOP2, with the exception of model 6 which recorded a slightly higher relative difference of $12.5 \%$.

Even though model 4 contains furniture layout as per Model 1, 2 and 3; the relative difference in RT recorded for model 4 in DOP2 and GOP2 still exceeded the maximum tolerance with each logged a $19.9 \%$ and $13 \%$ relative difference. All OPOs in Model 1 to 3 recorded an agreeable RT relative difference of fewer than $10 \%$.

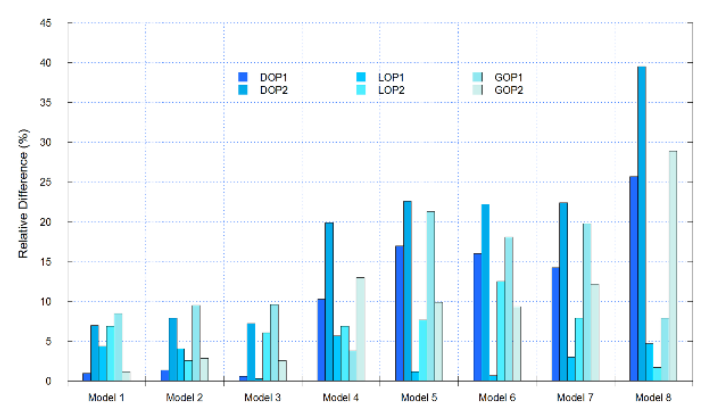

Figure 6: Comparison of the relative difference between all OPOs in all models

\subsection{Speech Transmission Index (STI)}

Speech transmission index (STI) is one of the key parameters in assessing the acoustical performance in OPOs. STI data simulated on all points will be utilized for comparison with the measured STI data. The $\mathrm{x}$-axis from each graph (see Fig. 7) represents the receiver points for its individual OPO and the y-axis as the STI value from the minimum value of 0 to the maximum value of 1 . The receiver points in the $\mathrm{x}$-axis were arranged so that the first point is the nearest from the sound source and the last point as the furthest. The comparison of STI between the measured and simulated for all models from all OPOs is presented in Fig. 7. The simulated STI from selected receiver points is compared directly with the measured STI data of the same receiver points.

The plot-patterns of STI data from all OPOs show a promising comparison. The STI data plotted from the receiver points seems to match similarly to the measured STI data. Also, it should be noted that STI data for model 1 to 4 plotted more closely with the measured data compared to model 5 to 8 , which has no furniture layout inside the models. Even though the plotpatterns show encouraging results, the discrepancies in STI values should not be dismissed. This is especially crucial when LOP2 graph shows a significant difference in STI value despite the matching plot-pattern recorded through the simulation work.

While measuring the comparison for RT was done through relative difference, the appropriate way to compare STI value is through its own JND. ODEON recommended that the JND for STI is equal to a 0.03 absolute difference in STI value. Wang et al. (2014) described those 0.03 absolute differences in STI value (the smallest detectable difference) as 1JND. However, Bradley, Reich, and Norcross (1999) said that a 0.03 change in STI would be imperceptible. It also added that any change in STI smaller than 0.1 would not be too noticeable.

Table 4 shows the minimum, maximum and average absolute difference between the measured and simulated STI data from each particular point. The JND of STI value from all models in DOP1, DOP2, LOP1 and GOP1 shows satisfying results of having the JND of not more than 4JND. The highest difference detected from this group is the maximum JND from model 8 of DOP2 which recorded a 0.10 absolute difference; which is still considered acceptable (J. S. Bradley, 1999).

Meantime, LOP2 and GOP2 recorded maximum JND of more than 4JND in model 5 to 8 . 


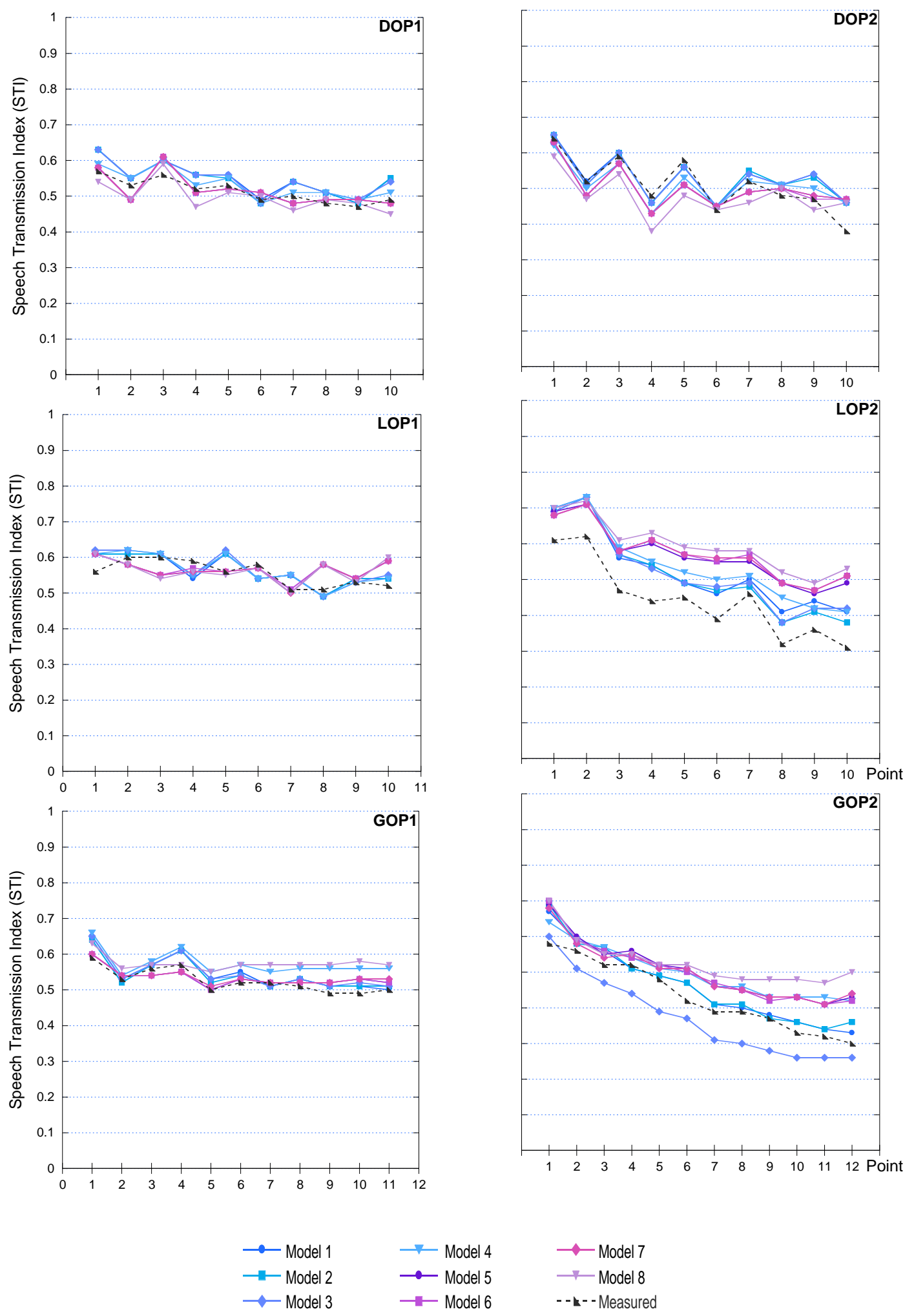

Figure 7: Comparison of measured and simulated STI data for all OPOs. 
Maximum JND value in models 1 to 4 recorded around 3JND and 4JND which is between 0.09 to 0.12 absolute differences, except for model 4 of LOP2 which recorded a JND of 0.13 .

\subsection{Spatial Decay in Sound Pressure Level (SPL)}

Similarly to the comparison made for STI, spatial decay is presented by plotting the SPL in measured and simulated points. The comparison of SPL decay is illustrated in Fig. 8. The $x$-axis represents the receiver points in which the measurement were taken and the $y$-axis is the sound pressure level in $\mathrm{dB}(\mathrm{A})$ taken at the said point. The measured SPL data were not actually presented in the previous work, but the measurements for the presented measured SPL in this study were made during the same sessions.

Table 4. The comparison of the minimum, maximum, and average JND value between the measured and simulated STI data for all OPOs

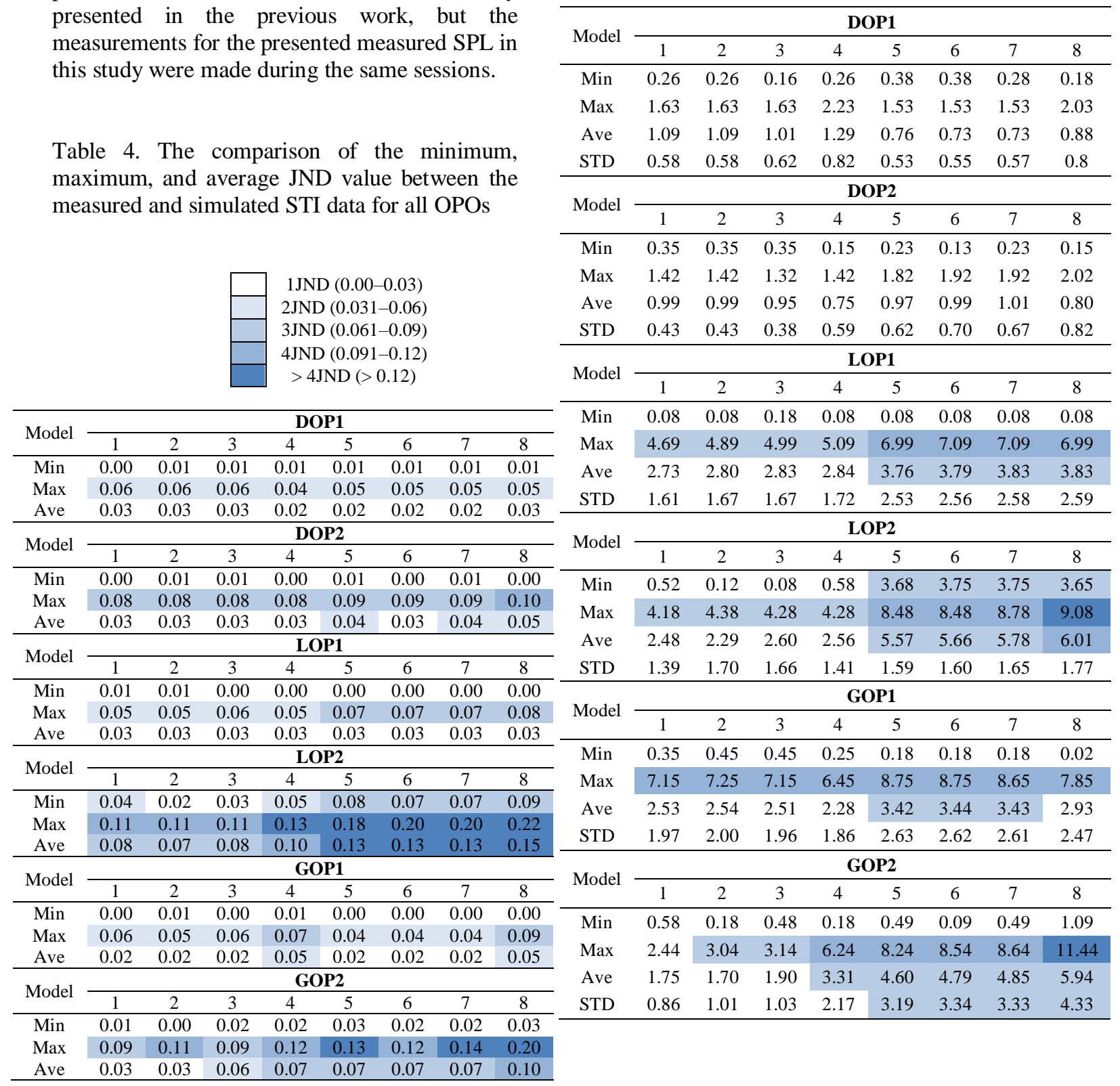

Table 5. The comparison of the minimum, maximum, average, and standard deviation difference value between the measured and simulated SPL data for all OPOs

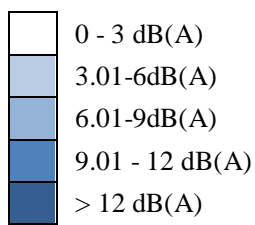


Comparison of SPL through the plot-pattern shows encouraging results in DOP1, DOP2, LOP2 and GOP2. The decay curve shows similar tendencies as the measured SPL data. Meanwhile, LOP1 and GOP1 plot-patterns show quite a disagreement as the measured SPL data resulted in random fluctuation at certain receiver points.

Considering $3 \mathrm{~dB}(\mathrm{~A})$ as the JND for SPL comparison (L. M. Wang et. al, 2004), Table 5 shows the minimum, maximum, average and the standard deviation of the difference between measured and simulated SPL data for all OPOs. DOP1 and DOP2 show good results as the difference in SPL data simulated do not exceed 3 $\mathrm{dB}(\mathrm{A})$ in all models. LOP1, LOP2, GOP1 and GOP2 show relatively agreeable results. While most of the maximum SPL differences recorded exceeded the limit of $3 \mathrm{~dB}(\mathrm{~A})$, model 1 to 4 in LOP1 and LOP2 does not exceed $6 \mathrm{~dB}(\mathrm{~A})$ difference. GOP1 and GOP2 however, recorded quiet excessive differences between the measured and simulated SPL data.

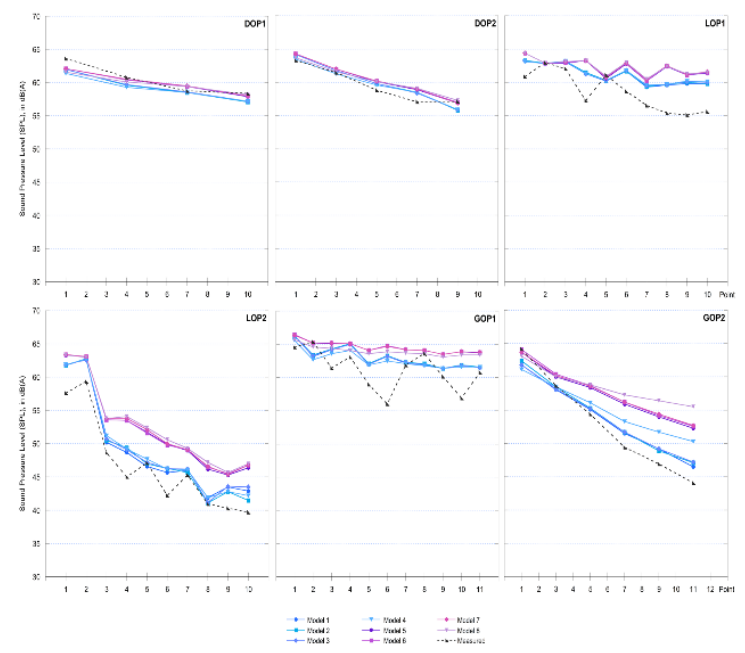

Fig. 8: Comparison of measured and simulated decay of SPL data for all OPOs.

\section{CONCLUSION}

A few important details were observed throughout the comparison and simulation process. The absorption coefficient of materials contributes to an important element in the simulation of reverberation time (RT). Small changes in the absorption coefficient in any materials, especially in materials with the large surface area could affect the RT results significantly. This explained the substantial changes in the RT relative difference between model 1 to 4 and model 5 to 8 . With the addition of furniture layout in the models, the RT improves considerably. It is safe to say that the sound energy absorbed by the furniture also contributes to achieving good RT results.

Meanwhile, for speech transmission index (STI), the background noise (BN) input is very essential. Without the $\mathrm{BN}$ input, reliable STI results are impossible to be achieved. However, it should be noted that while model 1 to 4 contain furniture layout and model 5 to 8 did not, the plot patterns of simulated STI and SPL data did not fluctuate too significantly from each other. However, noticeable change in value can be seen especially in the spatial decay of sound pressure level (SPL). It is observed that the SPL in models 1 to 4 (with furniture) decay more accurately with the measured SPL decay results.

In terms of model detail level, it was observed that models 1, 2 and 3 bear satisfactory simulated data when compared to their respective measured data. Pertaining to this finding, it was found that model simplification by up to $80 \%$ reduction in number of surfaces are acceptable. However, taking into consideration the differences in modelling technique of the person, the ambiguity of the model setup and settings in the simulation tools, and the accuracy of the materials' absorption coefficient and the scattering coefficient applied to the materials in the room; this reduction percentage might be marginally varied. Nevertheless, it is acceptable to say that as long as the room's major surfaces were presented, the simulation can bear agreeable results. However, it should also be noted that a too simplify room geometry would affect some acoustical parameters, as seen in models 4.

\section{REFERENCES}

J. Schroeder, K. Martin and B. Lorensen, The Visualization Toolkit. An Object-Oriented Approach to 3D Graphics, Prentice Hall, New Jersey, (1996).

A. Koutamanis, "Digital architectural visualization", Automation in Construction, 9(4), 347-360, (2000).

Ö. Akin, "Computational Design Instruction: Toward a Pedagogy", in Electronic design studio: architectural knowledge and media 
in the computer era, edited by $\mathrm{M}$. McCullough, W. J. Mitchell, and P. Purcell, MIT Press, (1990), Pages 301-316.

$\mathrm{X}$. Yin, P. Wonka and A. Razdan, "Generating 3D Building Models from Architectural Drawings: A Survey", IEEE Computer Graphics and Application, 29 (1), 20-30, (2009).

R. Oxman, "Digital architecture as a challenge for design pedagogy: theory, knowledge, models and medium", Design Studies, 29(2), 99-120, (2008).

A. Yezioro, B. Dong and F. Leite, "An applied artificial intelligence approach towards assessing building performance simulation tools", Energy and Buildings, 40, 612-620, (2008).

T. Hong, S. K. Chou and T. Y. Bong, "Building simulation: an overview of developments and information sources", Building and Environment, 35, 347-61, (2000).

D. J. Oldham and M. A. Rowell, "Computer Applications in Building and Environmental Acoustics", Building and Environment, 22(3), 189-200, (1987).

M. Hornikx, "Acoustic modelling for indoor and outdoor spaces", Journal of Building Performance Simulation, 8(1), 1-2, (2015).

J. L. M. Hensen and R. Lamberts, Building Performance Simulation for Design and Operation, Spon Press, London, UK, (2001).

J. L. M. Hensen, "Towards more effective use of building performance simulation in design", in Proc. 7th International Conference on Design \& Decision Support Systems in Architecture and Urban Planning, Eindhoven University of Technology, (2004).

G. Augenbroe, "Trends in building simulation", Building and Environment, 37, 891-902, (2002).

S. Siltanen, T. Lokki, L. Savioja and C. L. Christensen, "Geometry Reduction in Room Acoustics Modeling", Acta Acustica united with Acustica, 94, 410-418, (2008).

C. Andújar, D. Ayala and P. Brunet, The Discretized Polyhedra Simplification (DPS): a Framework for Polyhedra Simplification Based on Decomposition Schemes, Technical Report IRI-DT-99-00, Universitat Politècnica de Catalunya, Barcelona, (1999).

C. Andújar, P. Brunet and D. Ayala, "TopologyReducing Surface Simplification Using a
Solid Representation”, ACM Transactions on Graphics, 21(2), 88-105, (2002).

H. Kuttruff, Room Acoustics, Fifth Edition, Spon Press, Abingdon, Oxon, (2009).

T. He, L. Hong, A. Varshney and S. W. Wang, "Controlled Topology Simplification", IEEE Transactions on Visualization and Computer Graphics, 2(2), 171-184, (1996).

F. S. Nooruddin and G. Turk, "Simplification and Repair of Polygonal Models Using Volumetric Techniques", IEEE Transactions on Visualization and Computer Graphics, 9(2), 191-205, (2003).

J. H. Rindel and C. L. Christensen, "ODEON, A Design Tool for Noise Control in Indoor Environments", in Proceedings of the International Conference Noise at Work, (2007).

J. H. Rindel, "Room Acoustic Prediction Modelling", in XXIII Encontro Da Sociedade Brasileira de Acústica, Salvador, Brazil, (2010).

M. Vorländer, "Review Papers - Virtual Acoustics", Archives of Acoustics, 39(3), 307-18, (2014).

N. A. Abd Jalil, N. B. Che Din and N. Keumala, "Assessment on acoustical performance of green office buildings in Malaysia", Indoor and Built Environment, 25(4), 589-602, (2016).

S. A. Ali, "Open-plan office noise levels, annoyance and countermeasures in Egypt", Noise Control Engineering Journal, 59(2), 186-193, (2011).

S. Pelzer, M. Aretz and M. Vorländer, "Quality assessment o room acoustic simulation tools by comparing binaural measurements and simulations in an optimized test scenario", in Forum Acusticum Aalborg, 1529-1534, (2011).

J. H. Rindel, "The Use of Computer Modeling in Room Acoustics", Journal of Vibroengineering, 3(4), 219-24, (2000).

C. L. Christensen and G. Koutsouris, "ODEON Room Acoustics Software Version 12. Basic, Industrial, Auditorium and Combined Editions", Second Edition, ODEON A/S, Lyngby, (2013)

C. L. Christensen and J. H. Rindel, "A new scattering method that combines roughness and diffraction effects", Forum Acusticum Budapest, (2005).

ISO 3382-3:2012. "Acoustics - Measurement of room acoustic parameters. Part 3: Open plan offices". 
H. Shiokawa and J. H. Rindel, Comparison between Computer Simulations of Room Acoustical Parameters and Those Measured in Concert Halls, Report of the Research Institute of Industrial Technology, Nihon University, Tokyo, (2007).

N. A. Abd Jalil, N. B. Che Din and N. Keumala, "A Literature Analysis on Acoustical Environment in Green Building Design Strategies", Applied Mechanics and Materials, 471, 138-142, (2014).

G. A. Coudriet, "Acoustical challenges posed by sustainable building design", InterNoise 2009, (2009).

S. Abbaszadeh, L. Zagreus, D. Lehrer and C. Huizenga, Occupant Satisfaction with Indoor Environmental Quality in Green Buildings. Center for the Built Environment, UC Berkeley, (2006).

Y. S. Lee and S. K. Kim, "Indoor Environmental Quality in LEED-Certified Buildings in the U.S", Journal of Asian Architecture and Building Engineering, 7(2), 293-300, (2008).

R. T. Muehleisen, "Acoustics of Green Buildings", Implication, 8(1), 1-7, (2006).

Y. S. Lee, "Office layout affecting privacy, interaction, and acoustic quality in LEEDcertified buildings", Building and Environment, 45(7), 1594-1600, (2010).
X. Wang, Y. Koba, S. Ishikawa and S. Kijimoto, "Hybrid active noise barrier with sound masking in open-plan offices", Noise Control Engineering Journal, 64(3), 403514, (2016).

M. Hodgson, "When is Diffuse-Field Theory Applicable?", Applied Acoustics, 49(3), 197-207, (1996).

S. Bistafa and J. Bradley, "Predicting reverberation times in a simulated classroom", The Journal of the Acoustical Society of America, 108(4), 1721-1731, (2000).

J. Wang, N. Yan, D. Wang and J. Peng, "Comparison of Objective Acoustical Indices in Different Classroom Environments in Primary School", The 21st International Congress on Sound and Vibration, (2014).

J. S. Bradley, R. Reich and S. G. Norcross, "A just noticeable difference in C50 for speech", Applied Acoustics, 58, 99-108, (1999).

L. M. Wang and M. C. Vigeant, "Objective and Subjective Evaluation of the Use of Directional Sound Sources in Auralizations", in 18th International Congress on Acoustics, 2711-2714, (2004). 